\title{
Evaluation of two collagen conduits and autograft in rabbit sciatic nerve regeneration with quantitative magnetic resonance DTI, electrophysiology, and histology
}

Tina Jeon ${ }^{1 *}$, Emil S. Vutescu², Eliana B. Saltzman², Jordan C. Villa², Scott W. Wolfe ${ }^{2}$, Steve K. Lee ${ }^{2}$, Joseph H. Feinberg ${ }^{3}$, Sarah L. Pownder ${ }^{1}$, Jonathan P. Dyke ${ }^{4}$ and Darryl B. Sneag ${ }^{1}$

\begin{abstract}
Background: We compared different surgical techniques for nerve regeneration in a rabbit sciatic nerve gap model using magnetic resonance diffusion tensor imaging (DTI), electrophysiology, limb function, and histology.

Methods: A total of 24 male New Zealand white rabbits were randomized into three groups: autograft $(n=8)$, hollow conduit $(n=8)$, and collagen-filled conduit $(n=8)$. A 10-mm segment of the rabbit proximal sciatic nerve was cut, and autograft or collagen conduit was used to bridge the gap. DTI on a 3-T system was performed preoperatively and 13 weeks after surgery using the contralateral, nonoperated nerve as a control.

Results: Overall, autograft performed better compared with both conduit groups. Differences in axonal diameter were significant (autograft > hollow conduit > collagen-filled conduit) at 13 weeks (autograft vs. hollow conduit, $p=0.001$, and hollow conduit vs. collagen-filled conduit, $p<0.001$ ). Significant group differences were found for axial diffusivity but not for any of the other DTI metrics (autograft $>$ hollow conduit $>$ collagen-filled conduit) (autograft vs. hollow conduit, $p=0.001$ and hollow conduit vs. collagen-filled conduit, $p=0.021$ ). As compared with hollow conduit (autograft $>$ collagen-filled conduit > hollow conduit), collagen-filled conduit animals demonstrated a nonsignificant increased maximum tetanic force.

Conclusions: Autograft-treated rabbits demonstrated improved sciatic nerve regeneration compared with collagen-filled and hollow conduits as assessed by histologic, functional, and DTI parameters at 13 weeks.
\end{abstract}

Keywords: Trauma (nervous system), Nerve regeneration, Peripheral nerves, Sciatic nerve, Diffusion tensor imaging (DTI)

\section{Key points}

- Diffusion tensor imaging may be a biomarker for peripheral nerve regeneration

- Autograft shows improved sciatic nerve regeneration compared to collagen-based conduits

- Axial diffusivity correlated with measures of axonal diameter

\footnotetext{
* Correspondence: jeont@hss.edu

'Department of Radiology and Imaging, Hospital for Special Surgery, $535 \mathrm{E}$

70th Street, New York, NY 10021, USA

Full list of author information is available at the end of the article
}

\section{Background}

Traumatic peripheral nerve injury is a potentially devastating ailment resulting in severe sensory and/or motor deficits. Neurotmetic injury, characterized by complete disruption of the nerve and its supporting structures, usually requires surgical intervention. Peripheral nerve injuries that cannot be repaired by a direct end-to-end, tensionless technique are addressed using either nerve autograft, the current standard of care $[1,2]$, nerve allograft, or a nerve conduit to bridge the gap. However, the use of autograft involves inherent morbidity at the harvested donor site, including the 
potential for neuroma formation, as well as increased operative time and cost due to a second surgical dissection [3-5].

Several nerve conduit types are currently in use or under investigation, and two of them were evaluated in this study. A Food and Drug Administration-approved conduit, NeuraGen $^{\odot}$, (Integra LifeSciences, Plainsboro, New Jersey, USA; www.integralife.com) comprises a hollow inner structure and porous outer layer, both composed of a semipermeable type I collagen engineered to a specific pore size to allow for nutrient diffusion and nerve growth factor retention [6]. The other conduit studied was Nerbridge ${ }^{\mathrm{TM}}$ (Toyobo Co., LTD, Osaka, Japan; www.toyobo-global.com), an investigational product which comprises a coated collagen exterior with a porous polyglycolic acid (PGA) inner structure filled with collagen fibers. The inner structure composition of Nerbridge $^{\mathrm{Ts}}$, in theory, provides a scaffold to guide longitudinal propagation of regenerating nerve fibers [7].

Measurement of muscle recruitment with electrodiagnostic testing is the current standard of reference for assessing motor recovery following peripheral nerve injury and subsequent surgical intervention. It is operator-dependent and mildly invasive, however, requiring small needle placement into muscles. Diffusion tensor imaging (DTI) is a noninvasive magnetic resonance imaging (MRI) technique that has recently shown potential in quantifying peripheral nerve degeneration and regeneration in vivo by measuring the Brownian motion of water molecules $[8,9]$. DTI indices reveal structural and orientation information of peripheral nerves including fractional anisotropy (FA), reflecting the degree of diffusion anisotropy of water molecules along axons, and the apparent diffusion coefficient (ADC), or magnitude of diffusivity. While axial diffusivity (AD) is inferred to reflect axon density, radial diffusivity (RD) is inferred to reflect myelin integrity [10, 11]. Alterations in DTI-derived metrics are believed to reflect changes in peripheral nerve microstructure and overall 'nerve health' [12]. Recent studies have demonstrated good correlation between DTI and functional and histologic parameters in assessing nerve regeneration in rodent models $[8,13]$ and rabbit models [14-17].

The goal of this study was to compare the efficacy of nerve regeneration between autograft and two different types of nerve conduits, Nerbridge ${ }^{\mathrm{Ts}}$ and NeuraGen ${ }^{\oplus}$, using DTI, electrodiagnostic testing, limb function, and histopathologic measurements.

\section{Methods}

\section{Subjects}

All procedures were performed in accordance with the National Institutes of Health Guide for the Care and Use of Laboratory Animals, with the approval from our Institutional Animal Care and Use Committee. A total of 24 adult male New Zealand white rabbits (weight $3.0-3.5 \mathrm{~kg}$ ) were randomized into three surgical groups of eight rabbits each: autograft, hollow conduit $\left(\right.$ NeuraGen $\left.{ }^{\odot}\right)$, and collagen-filled conduit (Nerbridge ${ }^{\mathrm{Tm}}$ ).

\section{Surgical procedure}

The nerve-gap surgical procedure was modeled after the description of Shin et al. [18]. Rabbits were induced intramuscularly with general anesthesia. The sciatic nerve was exposed along the far proximal, posterior right thigh, and a $10-\mathrm{mm}$ nerve segment removed at the midpoint between a line drawn between the greater trochanter and ischial tuberosity. In the autograft group, the orientation of the $10-\mathrm{mm}$ nerve segment was reversed and placed as an interposition graft with 9-0 nylon epineurial sutures (Ethicon Inc., Somerville, NJ, USA). For the conduit groups, the nerve gap was bridged with one of the two conduits (Fig. 1). MRI, histological, and functional testing analysis was performed blind to the type of surgical procedure performed. Figure 2 summarizes the timeline of the experimental methods.

\section{MRI acquisition and postprocessing}

MRI of the bilateral thighs of the rabbits was performed in the prone position under the same anesthetic regimen as the surgical procedure, preoperatively (1-3 days before surgery) and postoperatively (13 weeks postsurgery), on a 3-T Siemens Magnetom Prisma unit (Siemens Healthcare, Erlangen, Germany) using an 8-channel flexible array coil positioned dorsally combined with 1-3 elements of a 32-channel spine array coil positioned ventrally under the thighs. A three-dimensional diffusion-weighted reversed fast imaging with steady state free precession sequence was acquired to localize the sciatic nerve, with the following technical parameters: echo time $2.72 \mathrm{~ms}$; repetition time $9.45 \mathrm{~ms}$; matrix $512 \times 512$; field of view $170 \times 170 \mathrm{~mm}$; slice thickness $0.9 \mathrm{~mm}$, without gap; diffusion moment $90 \mathrm{~s} / \mathrm{mm}^{2}$; slabs 96; bandwidth $465 \mathrm{~Hz} /$ pixel, acquisition time $8 \mathrm{~min} 5 \mathrm{~s}$; number of excitations 1. A single-shot echo planar imaging DTI sequence was acquired with the following technical parameters: sensitivity encoding reduction factor 2; field of view $130 \times 75 \mathrm{~mm}$; matrix $76 \times 44$; slice thickness $1.7 \mathrm{~mm}$, without gap; echo time/repetition time $=47 / 3400 \mathrm{~ms}$; number of excitations 10 ; gradient directions 20, b-values 0 and $700 \mathrm{~s} / \mathrm{mm}^{2}$; acquisition time $11 \mathrm{~min} 42 \mathrm{~s}$.

The DTI post-processing procedure is described in Fig. 3.

\section{Physiologic/functional testing}

The rabbits underwent a nonsurvival motor testing procedure under anesthesia at the 13-week time point. Measurements were made with respect to percentage of the nonoperated nerve. 


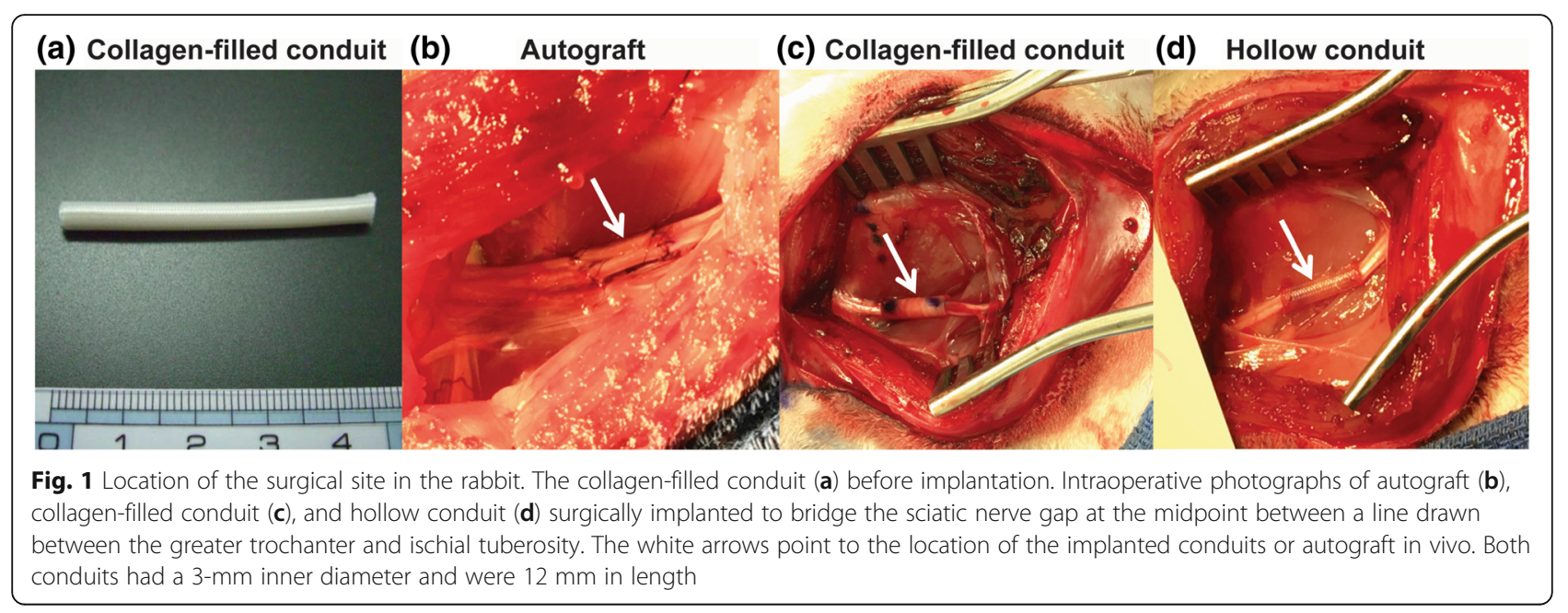

The animal weight was recorded immediately prior to surgery and prior to sacrifice. Anesthetized rabbits were examined for ankle contracture by measuring the angle between the anterior aspect of the tibia and dorsal aspect of the foot with the ankle in maximum passive plantar flexion.

To measure the compound motor action potential (CMAP), the sciatic nerve and its common peroneal and tibial divisions was exposed in the distal thigh and a miniature bipolar stimulating electrode needle was clamped on the exposed nerve. Recording electrodes were placed through the skin intramuscularly onto the surface of the tibialis anterior (TA) muscle and a ground surface electrode was placed on the foot for reference. Simulation duration was set at $0.02 \mathrm{~ms}$ and was performed at a stimulus intensity to obtain the maximum CMAP. CMAP latency and amplitude were measured using a Caldwell-Sierra EMG machine (Consolidated Neuro Supply Inc., Milford, OH, USA) with the Aurora Scientific software suite (Aurora, ON, USA; www.aurorascientific.com). The contralateral side was tested in an identical fashion.

The maximum isometric tetanic force (maximum tetanic) measurements were obtained to assess nerve potential force. The anesthetized rabbits were placed supine on a custom-built motor plate platform. A skin incision at the anterior aspect of the ankle was made to expose the TA muscle. The distal femur was stabilized to the platform by drilling into the distal femoral diaphysis (parallel to the plane of the tibial plafond) and stabilized with Kirschner wires (Pfizer Howmedica, Rutherford, NJ, USA). The distal TA tendon was secured to the level arm attached to the stimulator. The common peroneal nerve was then stimulated using a bipolar stimulator device (Harvard Apparatus, Holliston, MA, USA). For each muscle contraction, the electrical signal generated by the force transducer was processed using LabVIEW software (National Instruments, Austin, TX, USA). Maximum contractile force was calculated by subtracting the initial preload and repeated three times for each limb with a 5-min recovery interval.

At the conclusion of motor testing, all animals were sacrificed with $2.0 \mathrm{~mL}$ sodium pentobarbital $26 \%$ /isopropyl alcohol $10 \%$ administered through the lateral auricular vein.

The entire TA muscle from the operated and nonoperated limb was removed and weighed.

\section{Histopathological analysis}

Following animal sacrifice, 5-mm segments of nerve at the surgical site (proximal to the midpoint between a line drawn between the greater trochanter and ischial tuberosity) and distal to the surgical site ( $3 \mathrm{~mm}$ distal from

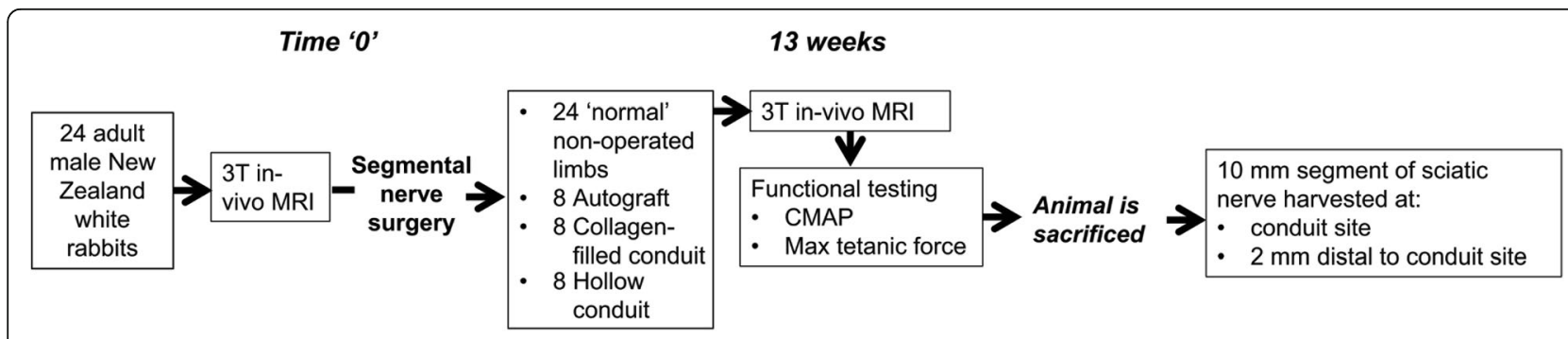

Fig. 2 Timeline and study design. CMAP compound motor action potential, MRI magnetic resonance imaging 


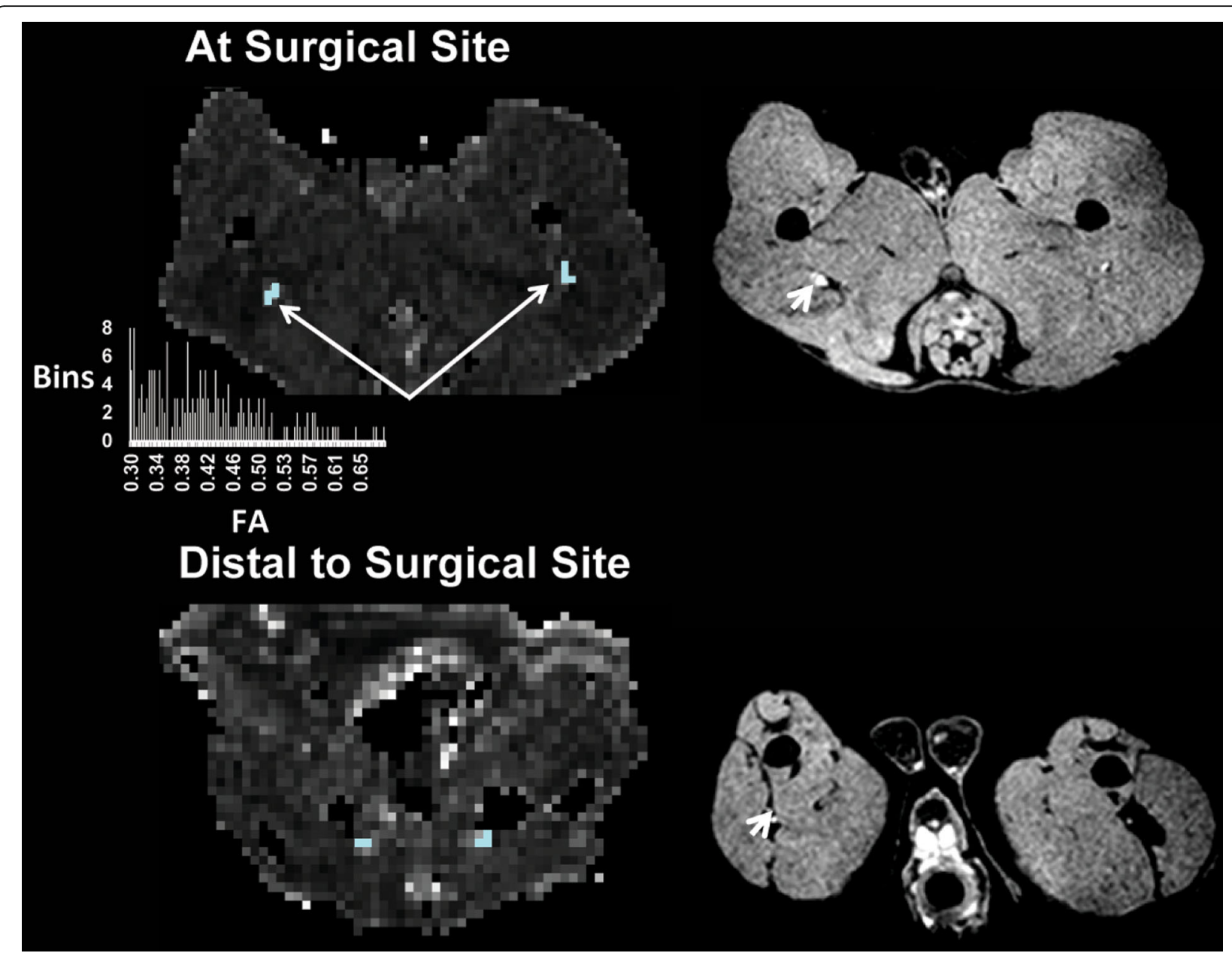

Fig. 3 Segmentation of the sciatic nerve. Tensor fitting was performed with DTI Studio (www.mristudio.org) to obtain DTI maps after motion and distortion correction by linearly registering all gradient directions to the $b=0$ map. Sciatic nerves were manually segmented using the fractional anisotropy ( $F A$ ) map (left panel) as a mask by thresholding the nerve (minimum, maximum $F A=0.3,0.8$ ) and using the reversed fast imaging with steady state free precession sequence for anatomical nerve localization (right panel). A region-growing algorithm, using a custom code written in Interactive Data Language (www.harrisgeospatial.com), was employed to segment the entire nerve length. DTI measurements were extracted from voxels between the 25th and the 75th percentiles of the FA histogram to mitigate partial volume effects at the peripheral margins of the nerve. The surgical site was located proximal to the midpoint between a line drawn between the greater trochanter and ischial tuberosity (right panel, white arrows). Similarly, the distal site was located $3 \mathrm{~mm}$ distal to the surgical site on the FA map. Only the voxels inside the mask were included in the analysis

this location) were harvested from the operated limb and at the same anatomic location from the nonoperated limb. Nerve samples were fixed in glutaraldehyde for $24 \mathrm{~h}$ and washed in phosphate-buffered saline (pH 7.4).

Samples were then cut into $1-\mathrm{mm}$ segments taken from the middle portion of each sample and postfixed in $1 \%$ osmium tetroxide for $6 \mathrm{~h}$. The samples were dehydrated through ethanol solution and soaked in propylene oxide. They were then embedded individually in epoxy resin to polymerize overnight. Excess epoxy resins surrounding the sample were trimmed and cut into semi-thin transverse sections of $2 \mu \mathrm{m}$ in thickness and stained with toluidine blue dye.
Transverse sections were viewed under a light microscope, and randomly sampled microscopic visual fields were captured using image analysis software (BIOQUANT Osteo II, BIOQUANT Image Analysis Corporation, Nashville, TN, USA) to quantify myelinated axonal density and diameter.

\section{Statistical analysis}

Spearman correlation coefficients between DTI measurements and histology, DTI and motor testing, and between motor testing and histology were calculated along with sample size and Fisher-approximated 95\% confidence interval, stratified by treatment group and site. A linear mixed-effects regression model was used 
to compare DTI metrics of the operated and nonoperated nerve as well as to measure between group differences for all three groups for histology, motor testing, and DTI. The Omnibus $F$ test and pairwise group comparisons were calculated with Bonferroni correction for multiple comparisons. Omnibus group comparisons were conducted with the Kruskal-Wallis test and pairwise group comparisons were analyzed with the Steel-Dwass-Critchlow-Fligner test [19]. An

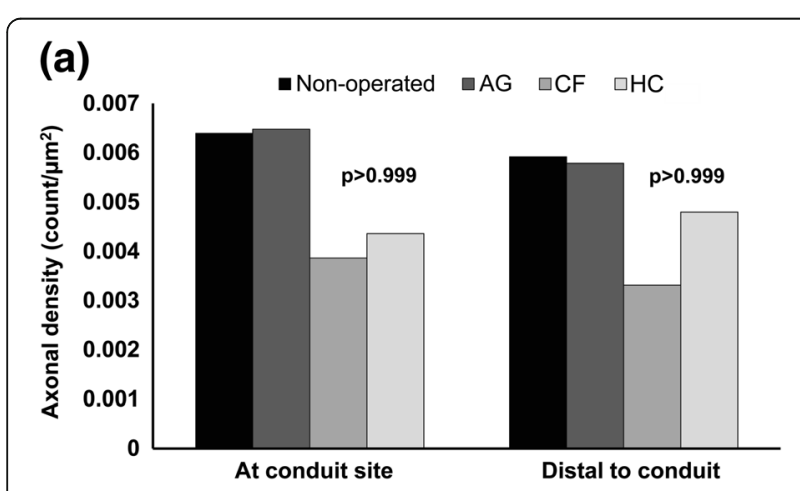

(b)
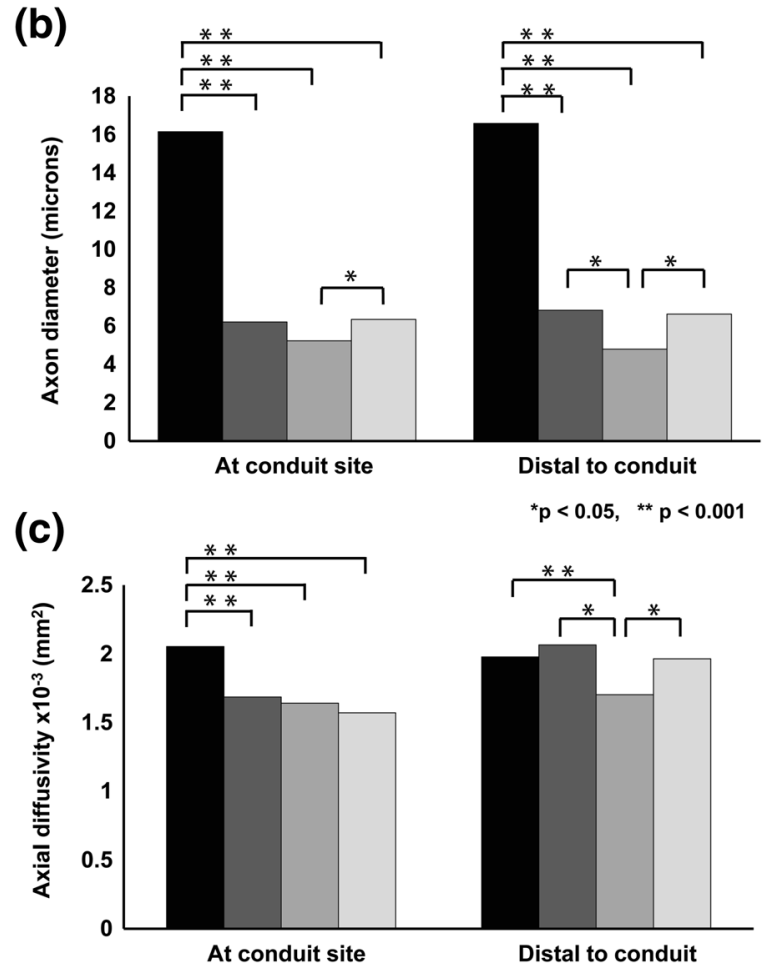

Fig. 4 Axon density, diameter, and axial diffusivity group differences at and distal to the surgical site. Myelinated axon density (a), diameter (b), and axial diffusivity (c) of the three different nerve surgeries, autograft $(A G)$, hollow conduit $(H C)$, and collagen-filled conduit (CF), compared with the nonoperated nerve. There were no significant differences between groups for axonal density either at or distal to the surgical site. The asterisks indicate statistical significance as indicated alpha value of $<0.05$ indicated significance, and all analyses were conducted in SAS 9.4 (SAS Institute Inc., Cary, NC, USA).

\section{Results}

Following the animal sacrifice, gross inspection of the surgical sites revealed prominent segments of scar tissue encasing the sciatic nerve for all groups. All non-nerve tissue was carefully discarded prior to histological examination. Two hollow conduits were still intact at 13 weeks while all of the collagen-filled conduits dissolved completely.

\section{Histopathologic findings}

Group differences were not statistically significant for axonal density (Fig. 4a). Significant differences in axonal diameter at the surgical site were found between the hollow conduit and collagen-filled conduit $(p=0.014)$. Distal to the surgical site, significant differences in axonal diameter between autograft and hollow conduit $(p=0.001)$ and hollow conduit and collagen-filled conduit $(p<0.001)$ were found (Fig. $4 \mathrm{~b})$. Representative histologic sections of the sciatic nerve for all groups are shown in Fig. 5.

\section{Physiologic/functional testing}

Differences in physiologic and functional measurements were not statistically significant between conduit groups (Table 1). Although not significant, the maximum tetanic force percentage increase (29.8\%) for the collagen-filled conduit was almost equivalent to that of autograft (35.3\%). However, there was high variance in both group measurements.

\section{DTI metrics}

Values for DTI metrics at 13 weeks are summarized in Table 2. FA, ADC, and RD were not significantly different between groups both at and distal to the surgical site. $\mathrm{AD}$ was significantly greater in autograft compared with collagen-filled conduit $(p=0.001)$ and greater in hollow conduit compared with collagen-filled conduit $(p=0.021)$ distal to the surgical site (Fig. 4c). AD group differences positively correlated with axonal diameter group differences between autograft and hollow conduit $(p=0.001)$ and between hollow conduit and collagen-filled conduit $(p<$ 0.001). Differences between DTI metrics of the nonoperated limb at the preoperative and postoperative time points were not significantly different for all rabbits irrespective of group $(p=0.751-0.909)$. DTI measurements of the entire length of the sciatic nerve revealed no significant group differences between all groups (Additional file 1: Figure S1).

There were moderate positive and negative correlations between axonal density and diameter and DTI metrics among all groups (Fig. 6a). The strongest 


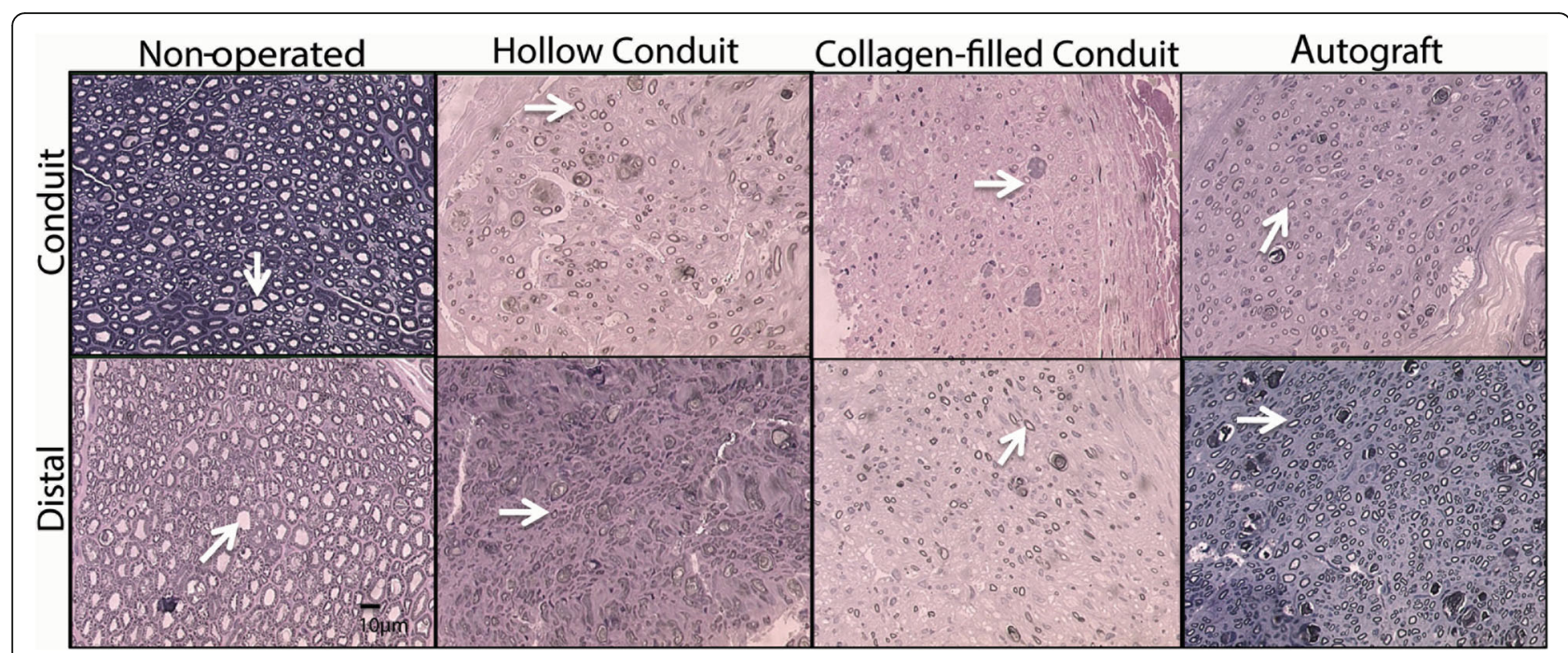

Fig. 5 Micrographs of orthogonal sections of the sciatic nerve 13 weeks after segmental nerve surgery. Histologic sections of the sciatic nerve distal to and at the surgical site 13 weeks postsurgery of autograft, collagen filled conduit, and hollow conduit with toluidine staining at 100x magnification. For comparison, the sciatic nerve of the nonoperated limb is shown in the left panel. The white arrows indicate myelinated axons. Note the visually decreased diameter of the operated nerves compared with normal controls. Due to processing difficulties, seven samples were not included in the histology analysis: two autograft at the conduit site, one autograft distal to the graft site, two collagen-filled at the conduit site, and two hollow conduit distal to the conduit site. Color differences are due to staining/drying of toluidine blue

positive correlations were found between $\mathrm{AD}$ and axonal diameter distal to the conduit site $(r=0.50)$, and for ADC and axonal diameter at the conduit site $(r=0.50)$. In addition, there were moderate correlations between functional measures and DTI metrics among all groups (Fig. 6b) and moderate to very strong negative correlations between functional measures and histology (Fig. 6c). Spearman's correlation coefficients were not significant between DTI and histology and DTI and functional measures.

\section{Discussion}

In our study, autograft facilitated the most sciatic nerve regeneration following segmental nerve surgery compared with collagen-based conduits. Significant group-level differences were only found with $\mathrm{AD}$ and axonal diameter distal to the surgical site, with autograft showing the greatest nerve regeneration, followed by hollow conduit and collagen-filled conduit, which may indicate that $\mathrm{AD}$ is potentially a viable DTI marker to detect group differences in axonal regeneration. Our results are corroborated by a prior ex-vivo rat study detecting a significant correlation of AD with axon diameter [13]. A possible explanation for this is the sensitivity of eigenvalues to underlying changes in axonal swelling and flow [14], which is abundant in axonal regeneration [15] and could have a direct impact on $\mathrm{AD}$. In theory, water diffusion parallel to the axon is decreased due to a restriction in flow during injury.
Previous studies have shown in-vivo DTI to be predictive of nerve degeneration and regeneration in rabbit $[16-18,20]$ and rat $[8-10]$ sciatic nerve crush and laceration injury models. These studies demonstrated an initial FA decrease and RD increase in the sciatic nerve distal to the injury site immediately following sciatic nerve crush injury, presumably due to Wallerian degeneration. Thereafter, there was a period of significant FA increase and $\mathrm{RD}$ decrease due to regenerating myelin sheath $[8,10,16-18,20]$ until reaching preinjury levels at 10 weeks. In our study, we did not find a significant $\mathrm{RD}$ decrease by week 13 , which may indicate that the regenerative phase was incomplete at this time. Previous animal studies have shown that FA and RD correlate with the ratio of myelinated axons and total number of myelinated axons [8], which concur with DTI degenerative studies in the brain [21,22].

Several animal model studies have shown that bioabsorbable nerve conduits are efficacious in facilitating regeneration across nerve gaps [23-28]. Prior studies examined the best combination of biomaterials to replicate autograft properties [29]. An ideal conduit should arguably include the presence of a basal lamina scaffold to serve as an adhesive and to promote dendrite elongation [30]. A semipermeable inner structure facilitates the entry of growth factors, secretion of neurotrophic factors by nerve stumps, and the exchange of metabolic nutrients [31]. The conduit should degrade at a rate long enough to warrant an environment in which 
Table 1 Physiological/functional testing measurements for all groups

\begin{tabular}{|c|c|c|c|}
\hline & Autograft & Hollow conduit & Collagen-filled conduit \\
\hline \multirow{3}{*}{ Animal weight } & $0.2 \pm 9.4 \%$ & $-7.4 \pm 8.5 \%$ & $-3.6 \pm 6.7 \%$ \\
\hline & \multicolumn{2}{|c|}{$p=0.212$} & $p=0.545$ \\
\hline & \multicolumn{3}{|c|}{$p=0.907$} \\
\hline \multirow{3}{*}{ Ankle contracture } & $70.1 \pm 18 \%$ & $87.8 \pm 13.7 \%$ & $85.8 \pm 15 \%$ \\
\hline & \multicolumn{2}{|c|}{$p=0.148$} & $p=0.879$ \\
\hline & \multicolumn{3}{|c|}{$p=0.250$} \\
\hline \multirow{3}{*}{ Muscle weight } & $49.4 \pm 9.1 \%$ & $43.5 \pm 6.3 \%$ & $37.9 \pm 11.4 \%$ \\
\hline & \multicolumn{2}{|c|}{$p=0.303$} & $p=0.479$ \\
\hline & \multicolumn{3}{|c|}{$p=0.089$} \\
\hline \multirow{3}{*}{$\begin{array}{l}\text { Compound motor } \\
\text { action potential }\end{array}$} & $59.1 \pm 37.0 \%$ & $59.8 \pm 35.4 \%$ & $47.8 \pm 37.3 \%$ \\
\hline & \multicolumn{2}{|c|}{$p=0.999$} & $p=0.861$ \\
\hline & \multicolumn{3}{|c|}{$p=0.732$} \\
\hline \multirow{3}{*}{ Maximum tetanic force } & $35.3 \pm 27.3 \%$ & $15.9 \pm 9.0 \%$ & $29.8 \pm 26.3 \%$ \\
\hline & \multicolumn{2}{|c|}{$p=0.213$} & $p=0.889$ \\
\hline & \multicolumn{3}{|c|}{$p=0.889$} \\
\hline
\end{tabular}

Data are reported as mean \pm standard deviation

Animal weight is reported as percentage weight loss/gain 13 weeks postoperatively

Ankle contracture, muscle weight, compound motor action potential, and maximum tetanic force are reported as percentage of the nonoperated nerve

All $p$ values were obtained according to the Steel-Dwass-Critchlow-Fligner method

regeneration and reorganization of the nerve is effective, but slow enough to deter scar tissue accumulation inhibiting longitudinal nerve growth [32, 33].

The Nerbridge ${ }^{\mathrm{TM}}$ collagen-filled conduit is a collagencoated PGA conduit and porous collagen scaffold inner tube (collagen types I and III). It is biocompatible and commercially available for use in Japan. A previous rat study of a 1-mm transected laryngeal nerve bridged either with the collagen-filled conduit or direct suture assessed vocal fold mobility, nerve conduction velocity, morphology, and histology at 15 weeks following transection [34]. No significant differences in vocal fold recovery or conduction velocity were found in either group. However, more clearly myelinated fibers and less laryngeal muscle atrophy were observed for the collagen-filled conduit, supporting its potential efficacy. As evidenced in prior studies using conduits composed of PGA (the material of the collagen-filled outer core of the conduit), the structure tended to resorb quicker than other synthetic conduits [23, 35], further evidenced by a fully dissolved collagen-filled conduit at 13 weeks postsurgery. PGA-based conduits were superior to autograft in sensory recovery assessments in nerve gaps of less than $4 \mathrm{~mm}$ and greater than $8 \mathrm{~mm}$ [36]. A similar study in a rodent model involving much larger cohort sizes (without MRI) demonstrated overall similar results at the 12-week time point; however, analysis of a later 16-week time point revealed an accelerated rate of recovery in axonal density for collagen-filled conduit between 12 and 16 weeks postoperatively [37]. This suggests that the collagen-filled conduit may show improved recovery over the course of a longer time point, which was not investigated here.

Several reasons may help explain significantly larger axonal diameter and $\mathrm{AD}$ in the hollow conduit compared with the collagen-filled conduit in our study. One 
Table 2 Diffusion tensor imaging metrics ( $F A, A D C, R D$, and $A D)$ for all groups at and distal to the surgical site

\begin{tabular}{|c|c|c|c|c|c|c|}
\hline & \multicolumn{3}{|c|}{ At surgical site } & \multicolumn{3}{|c|}{ Distal to surgical site } \\
\hline & Autograft & $\begin{array}{l}\text { Hollow } \\
\text { conduit }\end{array}$ & $\begin{array}{l}\text { Collagen- } \\
\text { filled } \\
\text { conduit }\end{array}$ & Autograft & $\begin{array}{l}\text { Hollow } \\
\text { conduit }\end{array}$ & $\begin{array}{l}\text { Collagen-filled } \\
\text { conduit }\end{array}$ \\
\hline \multirow{3}{*}{ FA } & $0.48 \pm 0.06$ & $0.50 \pm 0.10$ & \begin{tabular}{|l|}
$0.50 \pm 0.10$ \\
\end{tabular} & $0.45 \pm 0.09$ & $0.40 \pm 0.06$ & $0.40 \pm 0.04$ \\
\hline & \multicolumn{3}{|c|}{\begin{tabular}{l|l}
$p=0.999$ & \\
\end{tabular}} & \multicolumn{2}{|c|}{$p=0.999$} & $p=0.999$ \\
\hline & \multicolumn{3}{|c|}{$p=0.999$} & \multicolumn{3}{|c|}{$p=0.999$} \\
\hline \multirow{3}{*}{$\operatorname{ADC}\left(10^{-3} \mathrm{~mm}^{2} / \mathrm{s}\right)$} & $1.16 \pm 0.20$ & $1.10 \pm 0.18$ & $1.07 \pm 0.15$ & $1.32 \pm 0.31$ & $1.16 \pm 0.22$ & \multirow{2}{*}{$\begin{array}{c}1.36 \pm 0.13 \\
p=0.132\end{array}$} \\
\hline & \multicolumn{3}{|c|}{\begin{tabular}{l|l}
$p=0.999$ & \\
\end{tabular}} & \multicolumn{2}{|c|}{$p=0.296$} & \\
\hline & \multicolumn{3}{|c|}{$p=0.999$} & \multicolumn{3}{|c|}{$p=0.999$} \\
\hline \multirow{3}{*}{$\mathrm{RD}\left(10^{-3} \mathrm{~mm}^{2}\right)$} & $0.86 \pm 0.20$ & $0.82 \pm 0.18$ & $0.80 \pm 0.16$ & $1.08 \pm 0.19$ & $0.97 \pm 0.17$ & $1.08 \pm 0.13$ \\
\hline & \multicolumn{2}{|c|}{\begin{tabular}{l|l}
$p=0.999$ & \\
\end{tabular}} & $p=0.999$ & \multicolumn{2}{|c|}{\begin{tabular}{l|l}
$p=0.86$ & \\
\end{tabular}} & $p=0.83$ \\
\hline & \multicolumn{3}{|c|}{$p=0.999$} & \multicolumn{3}{|c|}{$p=1.0$} \\
\hline \multirow{3}{*}{$\mathrm{AD}\left(10^{-3} \mathrm{~mm}^{2}\right)$} & $1.69 \pm 0.39$ & $1.64 \pm 0.22$ & $1.57 \pm 0.15$ & $2.07 \pm 0.42$ & $1.97 \pm 0.12$ & $1.70 \pm 0.28$ \\
\hline & \multicolumn{2}{|c|}{\begin{tabular}{l|l}
$p=0.999$ \\
\end{tabular}} & $p=0.999$ & \multirow{2}{*}{\multicolumn{2}{|c|}{\begin{tabular}{l|l}
$p=0.891$ & \\
\end{tabular}}} & $p=0.021^{*}$ \\
\hline & \multicolumn{3}{|c|}{$p=0.711$} & & & $p=0.001^{*}$ \\
\hline
\end{tabular}

Data are reported as mean \pm standard deviation

All $p$ values were obtained with a pairwise $t$ test

$A D$ axial diffusivity, $A D C$ apparent diffusion coefficient, $F A$ fractional anisotropy, $R D$ radial diffusivity

* Statistical significance after Bonferroni correction

of the theoretical benefits of nerve conduits is to impede negative growth factors (e.g., excessive scar tissue, edema, inflammation, necrosis) from the internal, regenerating environment. Faster degradation rates of the collagen-filled conduit may be detrimental to nerve regeneration, as evidenced by the two hollow conduits still intact by week 13 compared with the collagen-filled conduit, all of which had dissolved. This could perhaps be specific to rabbits, due to their reliance on hopping and hind limb muscle function [38] which may result in proportionally larger amounts of scar tissue generated, although results of various transplants and regenerative successes in a rabbit and rat model were similar [39]. Rates of degradation as well as internal matrix morphology and pore size of conduits are areas that may warrant further exploration.

The extent of motor functional recovery was assessed by the change in maximum tetanic, CMAP, animal weight, ankle contracture, and muscle weight at the 13-week time point in the current study. Maximum tetanic is considered the most objective indicator of strength associated with the degree of muscle innervation by the nerve [40]. (a)

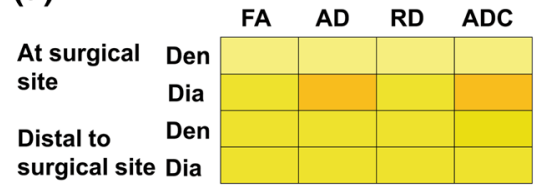

(c)
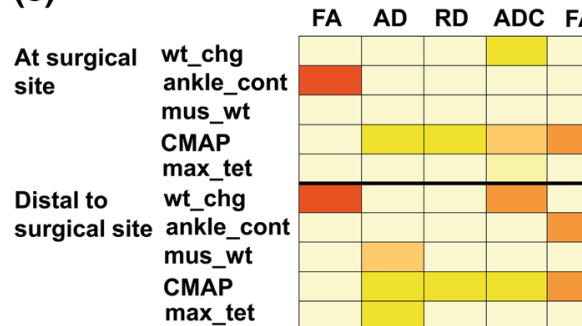

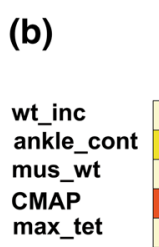

Collagen-

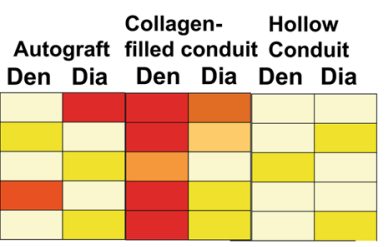

max_te

Autograft Collagen-filled conduit Hollow Conduit

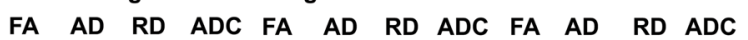

Legend

0-0.39

0.4-0.49

$0.5-0.59$

$0.6-0.69$

$0.7-0.79$

$0.8-1$

Fig. 6 Correlation between DTI, functional measures, and histology. Spearman correlations of DTI and myelinated axon density and diameter (a), between histology and functional measures (b), and between DTI and functional measures (c) of the three groups. The legend (right panel) indicates the level of correlation, defined as weak (0.0-0.39), moderate (0.40-0.59), strong (0.60-0.79), or very strong (0.80-1.0). AD axial diffusivity, ADC apparent diffusion coefficient, ankle_cont ankle contracture, CMAP compound motor action potential, Den axonal density, Dia axonal diameter, FA fractional anisotropy, max_tet maximum tetanic force, mus_wt muscle weight, RD radial diffusivity, wt_chg weight change 
However, in this investigation, tetanic contraction had a high variance but did show favorability towards the collagen-filled conduit and conflicted with AD and axonal diameter findings. Tetanic-induced contractions provide a purely functional response, which could be originating from smaller axons if they form a tighter connection with muscle tissue. Hence, axon diameter may not accurately reflect actual function.

Several limitations to this study should be considered. First, due to the small diameter of the rabbit sciatic nerve (approximately $2-3 \mathrm{~mm}$ ) and a relatively low in-plane spatial resolution of the echo-planar sequence $(1.7 \mathrm{~mm})$, partial volume effects at the borders of the nerve may have biased DTI measurements by averaging of voxels that include both nerve and surrounding scar tissue/muscle and may have falsely elevated ADC and lowered FA. Second, the study could have benefitted from the inclusion of more rabbits in each surgical group due to borderline $p$ values after Bonferroni correction. Third, unlike previous studies, our investigation did not evaluate continuous time points, but rather a single time point that we thought would afford enough time for significant axonal regeneration based on previous crush and laceration studies demonstrating nearly complete sciatic nerve remyelination for a rabbit sciatic nerve by weeks $8-10$ [16-18]. In our study, axon density and diameter did not return to expected levels as compared with the nonoperated nerve by the 13-week time point, indicating the healing process is likely longer for nerve gap versus laceration/crush injuries. It may be prudent in future studies to examine multiple time points following segmental nerve surgery and at a longer follow-up time to accurately determine the longitudinal time course of nerve regeneration with MRI, histology, and functional testing. Fourth, CMAP and maximum tetanic force are commonly used in research studies measuring functional recovery in rats [26, 27, 41], but we are unaware of such studies in rabbits. Additional functional measurements including the Tarlov scale, which assesses locomotor ability [42], and the toe-spreading reflex, which assess the degree of toe spread [43], have been evaluated in other rabbit studies $[16-18,20]$ and may have been useful to assess functional recovery.

In summary, autograft facilitated greater sciatic nerve regeneration following segmental nerve surgery compared with collagen-based conduits, hollow conduit, and collagen-filled conduit. Hollow conduit performed significantly better with regards to the axonal diameter and the DTI parameter of axial diffusivity compared with collagen-filled conduit. However, collagen-filled conduit performed better with regards to the maximum tetanic force, which is the most objective measure of functionality. This investigation suggests that DTI may be a potential, noninvasive biomarker to assess peripheral nerve regeneration. Ultimately, larger studies will need to be conducted to determine its efficacy and role.

\section{Additional file}

Additional file 1: Figure S1. Whole nerve mean DTI measurements. There are no significant DTI differences between autograft (AG), collagenfilled (CF), and hollow conduit $(\mathrm{HC})$, when measuring the proximal and distal sciatic nerve. (DOCX $2714 \mathrm{~kb}$ )

\section{Abbreviations}

AD: Axial diffusivity; ADC: Apparent diffusion coefficient; CMAP: Compound motor action potential; DTI: Diffusion tensor imaging; FA: Fractional anisotropy; MRI: Magnetic resonance imaging; PGA: Polyglycolic acid; RD: Radial diffusivity; TA: Tibialis anterior

Availability of data and materials

The data will not be shared because Toyobo Co. Ltd. wishes to not have the data published publicly.

\section{Funding}

This study was funded by Toyobo Co. Ltd.

\section{Acknowledgements}

The study was funded by a grant provided by Toyobo Co., Ltd. DBS, of the Hospital for Special Surgery, is the guarantor of this study. All procedures were performed in accordance with the NIH Guide for the Care and Use of Laboratory Animals with approval from our Institutional Animal Care and Use Committee at Weill Cornell Medical College. We thank Francisco Colon, LVT, LATG, and Vanessa Nieves, LVT, LATG, SRA, of the Research Animal Resource Center, Weill Cornell Medical Center, for their assistance in rabbit handling and Fred Xavier, MD, PhD, of the Department of Orthopedic Surgery, Hospital for Special Surgery, for his assistance in the rabbit surgeries. We greatly appreciate the statistical assistance of Bin Lin, MS, of the Healthcare Research Institute, Hospital for Special Surgery.

\section{Authors' contributions}

TJ collected the data and wrote the manuscript. DBS obtained funding for the project, designed the study, oversaw the investigation, and edited the manuscript. SWW and SKL provided surgical consultation for the study, designed the study, and edited the manuscript. JHF provided electrodiagnostic consultation for the study, designed the study, and edited the manuscript. ESV collected the data and edited the manuscript. EBS assisted with the rabbit surgeries and designed the study. JCV performed the rabbit surgeries and designed the study. SLP provided veterinarian consultation and edited the manuscript. JPD provided consultation for the imaging and edited the manuscript. All authors read and approved the final manuscript.

\section{Ethics approval}

This study was approved by the Institutional Animal Care and Use Committee at Weill Cornell Medical College.

Consent for publication

Not applicable

\section{Competing interests}

The authors of this manuscript declare relationships with Toyobo Co. Ltd.

\section{Publisher's Note}

Springer Nature remains neutral with regard to jurisdictional claims in published maps and institutional affiliations.

\section{Author details}

${ }^{1}$ Department of Radiology and Imaging, Hospital for Special Surgery, 535 E 70th Street, New York, NY 10021, USA. ${ }^{2}$ Department of Hand and Upper Extremity Service, Hospital for Special Surgery, 535 E 70th Street, New York, 
NY 10021, USA. ${ }^{3}$ Department of Rehabilitation Medicine, Hospital for Special Surgery, 535 E 70th Street, New York, NY 10021, USA. ${ }^{4}$ Citigroup Biomedical Imaging Center, Weill Cornell Medical College, 516 E 72nd Street, New York, NY 10021, USA.

\section{Received: 16 February 2018 Accepted: 24 May 2018}

Published online: 08 August 2018

\section{References}

1. Siemionow M, Brzezicki G (2009) Chapter 8. Current techniques and concepts in peripheral nerve repair. Int Rev Neurobiol 87:141-172

2. Ray WZ, Mackinnon SE (2010) Management of nerve gaps: autografts, allografts, nerve transfers, and end-to-side neurorrhapy. Exp Neurol 223:77-85

3. Belkas JS, Shoichet MS, Midha R (2004) Peripheral nerve regeneration through guidance tubes. Neurol Res 26:151-160

4. Battiston B, Geuna S, Ferrero M, Tos P (2005) Nerve repair by means of tubulization: literature review and personal clinical experience comparing biological and synthetic conduits for sensory nerve repair. Microsurgery 25:258-267

5. IJpma FFA, Nicolai J-PA, Meek MF (2006) Sural nerve donor-site morbidity. Ann Plast Surg 57:391-395

6. Dellon AL, Mackinnon SE (1988) An alternative to the classical nerve graft for the management of the short nerve gap. Plast Reconstr Surg 82:849-856

7. Matsui Y, Yamasaki T, Inoue T, Kamba T, Ogawa O (2015) Cavernous nerve graft reconstruction with a novel artificial conduit during robot-assisted laparoscopic radical prostatectomy. Open J Urol 5:118-122

8. Morisaki S, Kawai Y, Umeda M et al (2011) In vivo assessment of peripheral nerve regeneration by diffusion tensor imaging. J Magn Reson Imaging 33:535-542

9. Boyer RB, Kelm ND, Riley DC et al (2015) 4.7-T diffusion tensor imaging of acute traumatic peripheral nerve injury. Neurosurg Focus 39:E9

10. Lehmann HC, Zhang J, Mori S, Sheikh KA (2010) Diffusion tensor imaging to assess axonal regeneration in peripheral nerves. Exp Neurol 223:238-244

11. Zhou Y, Kumaravel M, Patel VS, Sheikh KA, Narayana PA (2012) Diffusion tensor imaging of forearm nerves in humans. J Magn Reson Imaging 36:920-927

12. Mori S, Zhang J (2006) Principles of diffusion tensor imaging and its applications to basic neuroscience research. Neuron 51:527-539

13. Takagi T, Nakamura M, Yamada M et al (2009) Visualization of peripheral nerve degeneration and regeneration: monitoring with diffusion tensor tractography. Neuroimage 44:884-892

14. Ford JC, Hackney DB, Alsop DC et al (1994) MRI characterization of diffusion coefficients in a rat spinal cord injury model. Magn Reson Med 31:488-494

15. Markus A, Patel TD, Snider WD (2002) Neurotrophic factors and axonal growth. Curr Opin Neurobiol 12:523-531

16. Li X, Chen J, Hong G et al (2013) In vivo DTI longitudinal measurements of acute sciatic nerve traction injury and the association with pathological and functional changes. Eur J Radiol 82:e707-e714

17. Sun C, Hou Z, Hong G, Wan Q, Li X (2014) In vivo evaluation of sciatic nerve crush injury using diffusion tensor imaging: correlation with nerve function and histology. J Comput Assist Tomogr 38:790-796

18. Shin RH, Friedrich PF, Crum BA, Bishop AT, Shin AY (2009) Treatment of a segmental nerve defect in the rat with use of bioabsorbable synthetic nerve conduits: a comparison of commercially available conduits. J Bone Joint Surg Am 91:2194-2204

19. Critchlow DE, Fligner MA (1990) On distribution-free multiple comparisons in the one-way analysis of variance. Comm State Theory Methods 20:127-139

20. Wan Q, Wang S, Zhou J et al (2016) Evaluation of radiation-induced peripheral nerve injury in rabbits with MR neurography using diffusion tensor imaging and T2 measurements: correlation with histological and functional changes. J Magn Reson Imaging 43:1492-1499

21. Wang S, Wu EX, Tam CN, Lau HF, Cheung PT, Khong PL (2008) Characterization of white matter injury in a hypoxic-ischemic neonatal rat model by diffusion tensor MRI. Stroke 39:2348-2353

22. Budde MD, Kim JH, Liang H, Russell JH, Cross AH, Song S (2008) Axonal injury detected by in vivo DTI correlates with neurological disability in a mouse model of multiple sclerosis. NMR Biomed 21:589-597

23. Yamasaki T, Fujiwara $H$, Oda R et al (2015) In vivo evaluation of rabbit sciatic nerve regeneration with diffusion tensor imaging (DTI): correlations with histology and behavior. Magn Reson Imaging 33:95-101
24. Evans GRD, Brandt K, Widmer MS et al (1999) In vivo evaluation of poly(L-lactic acid) porous conduits for peripheral nerve regeneration. Biomaterials 20:1109-1115

25. Azhar MM, Sara TA (2004) Comparison of nerve graft and artificial conduits for bridging nerve defects. Med J Malaysia 59:578-584

26. Waitayawinyu T, Parisi DM, Miller B et al (2007) A comparison of polyglycolic acid versus type 1 collagen bioabsorbable nerve conduits in a rat model: an alternative to autografting. J Hand Surg Am 32:1521-1529

27. Giusti G, Willems WF, Kremer T, Friedrich PF, Bishop AT, Shin AY (2012) Return of motor function after segmental nerve loss in a rat model: comparison of autogenous nerve graft, collagen conduit, and processed allograft (AxoGen). J Bone Joint Surg Am 94:410-417

28. Sahakyants T, Lee JY, Friedrich PF, Bishop AT, Shin AY (2013) Return of motor function after repair of a 3-cm gap in a rabbit peroneal nerve: a comparison of autograft, collagen conduit, and conduit filled with collagenGAG matrix. J Bone Joint Surg Am 95:1952-1958

29. Ichihara S, Inada Y, Nakamura T (2008) Artificial nerve tubes and their application for repair of peripheral nerve injury: an update of current concepts. Injury 39(Suppl 4):29-39

30. Longo FM, Hayman EG, Davis GE et al (1984) Neurite-promoting factors and extracellular matrix components accumulating in vivo within nerve regeneration chambers. Brain Res 309:105-117

31. Aebischer P, Guénard V, Winn SR, Valentini RF, Galletti PM (1988) Blind-ended semipermeable guidance channels support peripheral nerve regeneration in the absence of a distal nerve stump. Brain Res 454:179-187

32. Nicoli AN, Caliceti P, Lora S et al (1998) Polymer biomaterials (polyphosphazenes) in the repair of peripheral nervous system. Ann Ital Chir 67:843-848

33. Rodríguez FJ, Gómez N, Perego G, Navarro X (1999) Highly permeable polylactide-caprolactone nerve guides enhance peripheral nerve regeneration through long gaps. Biomaterials 20:1489-1500

34. Suzuki H, Araki K, Matsui T et al (2016) Value of a novel PGA-collagen tube on recurrent laryngeal nerve regeneration in a rat model. Laryngoscope 126:E233-E239

35. Clavijo-Alvarez JA, Nguyen VT, Santiago LY, Doctor JS, Lee WPA, Marra KG (2007) Comparison of biodegradable conduits within aged rat sciatic nerve defects. Plast Reconstr Surg 119:1839-1851

36. Weber RA, Breidenbach WC, Brown RE, Jabaley ME, Mass DP (2000) A randomized prospective study of polyglycolic acid conduits for digital nerve reconstruction in humans. Plast Reconstr Surg 106:1036-1045

37. Saltzman E, Villa JC, Feinberg JH, Lee SK, Wolfe SW (2017) Comparison between two collagen neve conduits and nerve autograft for motor nerve regeneration in a rat model. American Association for Hand Surgery Annual Meeting, Waikoloa, p 116 http://meeting.handsurgery.org/abstracts/2017-program.cgi

38. Gulati AK, Cole GP (1994) Immunogenicity and regenerative potential of acellular nerve allografts to repair peripheral nerve in rats and rabbits. Acta Neurochir (Wien) 126:158-164

39. Angius D, Wang H, Spinner RJ, Gutierrez-Cotto Y, Yaszemski MJ, Windebank AJ (2012) A systematic review of animal models used to study nerve regeneration in tissue-engineered scaffolds. Biomaterials 33:8034-8039

40. Swanson AN, Wolfe SW, Khazzam M, Feinberg J, Ehteshami J, Doty S (2008) Comparison of neurotization versus nerve repair in an animal model of chronically denervated muscle. J Hand Surg Am 33:1093-1099

41. Lee SK, Wolfe SW (2012) Nerve transfers for upper extremity: new horizons in nerve reconstruction. J Am Acad Orthop Surg 20:506-517

42. Varejão ASP, Cabrita AM, Meek MF et al (2004) Functional and morphological assessment of a standardized rat sciatic nerve crush injury with a non-serrated clamp. J Neurotrauma 21:1652-1670

43. Schmitz HC, Beer GM (2001) The toe-spreading reflex of the rabbit revisited_functional evaluation of complete peroneal nerve lesions. Lab Anim 35:340-345 